\title{
Central Molecular Zone of the Milky Way: Star Formation in an extreme Environment $\dagger$
}

\author{
Jens Kauffmann \\ Max-Planck-Institut für Radioastronomie, \\ Auf dem Hügel 69, D-53121 Bonn, Germany \\ email: jens.kauffmann@gmail.com
}

\begin{abstract}
The Central Molecular Zone (CMZ; inner 100 pc) hosts some of the most dense and massive molecular clouds of the Milky Way. These clouds might serve as local templates for dense clouds seen in nearby starburst galaxies or in the early universe. The clouds have a striking feature: they form stars at a very slow pace, considering their mass and high average density. Here we use interferometer data from ALMA and the SMA to show that this slow star formation is a consequence of the cloud density structure: CMZ clouds have a very flat density structure. They might, for example, exceed the average density of the Orion A molecular cloud by an order of magnitude on spatial scales $\sim 5 \mathrm{pc}$, but CMZ "cores" of $\sim 0.1 \mathrm{pc}$ radius have masses and densities lower than what is found in the Orion KL region. This absence of highest-density gas probably explains the suppression of star formation. The clouds are relatively turbulent, and ALMA observations of $\mathrm{H}_{2} \mathrm{CO}$ and $\mathrm{SiO}$ indicate that the turbulence is induced by high-velocity shocks. We speculate that these shocks might prevent the formation of high-mass cores. It has been argued that the state of CMZ clouds depends on their position along the orbit around Sgr $\mathrm{A}^{*}$. Our incomplete data indicate no evolution in the density structure, and only a modest evolution in star formation activity per unit mass.
\end{abstract}

Keywords. stars: formation, ISM: clouds, Galaxy: center

\section{Introduction: Dense Clouds with little Star Formation}

The Central Molecular Zone (CMZ) - i.e. the inner 100 pc of our Galaxy is a star-forming (SF) environment with very extreme physical properties. The CMZ produces 5 to $10 \%$ of the Milky Way's infrared and Lyman continuum luminosity, and $\sim 10 \%$ of its total molecular gas reside here. The molecular clouds have unusually high average densities $\gtrsim 10^{4} \mathrm{~cm}^{-3}$, they are closely packed, and they are subject to an average pressure (from X-ray data) of order $10^{6} \mathrm{~K} \mathrm{~cm}^{-3}$ (Morris \& Serabyn 1996, for all of the above). Using dust emission maps from Herschel, Molinari et al. (2011) recently showed that most of the gas in the CMZ resides on an orbit of about $100 \mathrm{pc}$ radius that has a complex geometry (Kruijssen et al. 2014).

It is important to study SF under these extreme conditions. First, here we have a unique chance to explore an extreme point in the SF parameter space. Comprehensive models should describe SF both in the CMZ as well as the solar neighborhood. Second, the CMZ clouds might serve as local templates for the types of clouds seen in nearby starbursts and the early universe. For example, the CMZ clouds have high average $\mathrm{H}_{2}$ column densities $\sim 10^{23} \mathrm{~cm}^{-2}$. These resemble values found in nearby starburst galaxies like the Antennae and NGC 253.

One particularly striking feature of CMZ molecular clouds is that star formation is suppressed. For example, Guesten \& Downes (1983) found that very few $\mathrm{H}_{2} \mathrm{O}$ masers

$\dagger$ with key contributions from T. Pillai, P. Goldsmith, Q. Zhang, X. Lu, A. Ginsburg, K. Immer, and A. Guzman 
(a) mass-size data

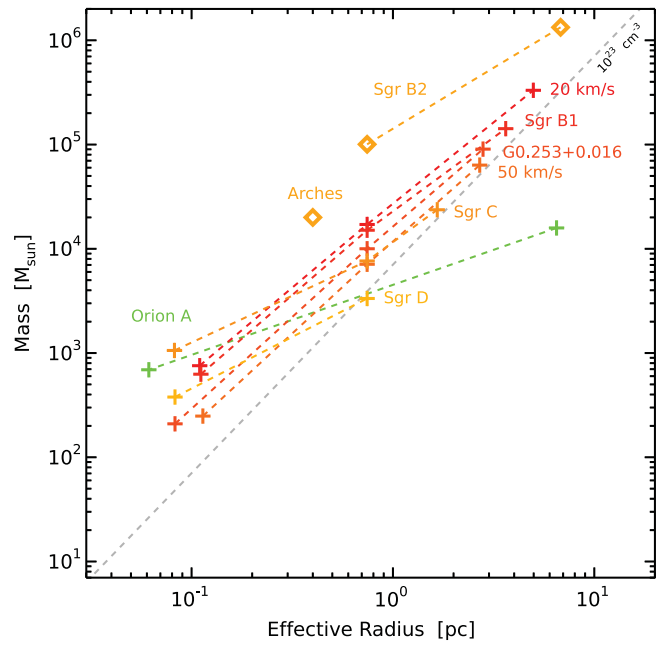

(b) mass-size slopes

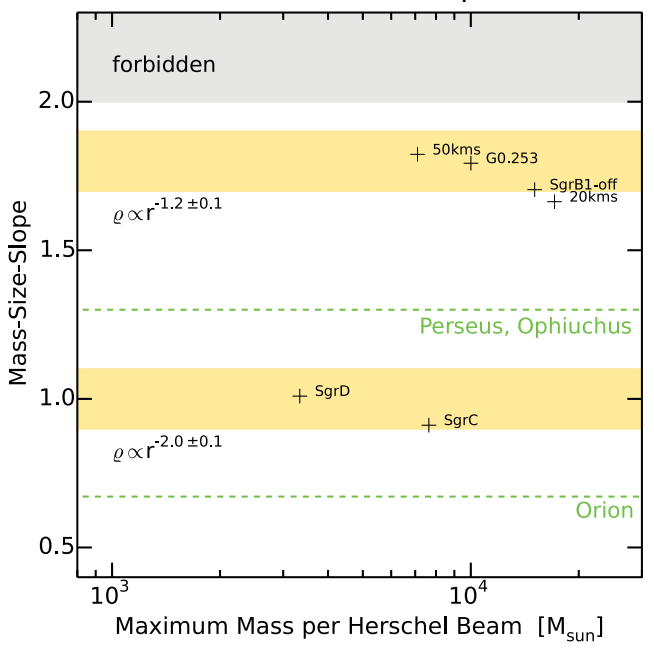

Figure 1. Mass-size analysis for CMZ clouds. (A Color version is available online.)

reside in the CMZ, relative to the high mass of dense gas. Longmore et al. (2012) provide an updated discussion of this trend: averaged over the entire CMZ star formation is suppressed by at least an order of magnitude, when compared to the solar neighborhood. Lis et al. (1994) and Kauffmann et al. (2013) demonstrate that SF is also suppressed on the scale of individual clouds.

In Kauffmann et al. (2013) we demonstrated that in one CMZ cloud, G0.253+0.016, the absence of significant SF can be explained by the absence of high-mass cores of $\sim 0.1 \mathrm{pc}$ size that could form stars efficiently. Here we show that this is a general feature of CMZ molecular clouds (Sec. 2) that is potentially induced by high-velocity shocks (Sec. 3). We explore the SF activity within the CMZ (Sec. 4) and find little evolution of cloud density structure and SF activity along the orbit of CMZ clouds (Sec. 5).

\section{Cloud Density Structure}

We conduct a mass-size analysis for the CMZ clouds to explore the density structure of our target clouds. The cloud structure on spatial scales $\gtrsim 1 \mathrm{pc}$ is investigated using Herschel-based dust emission maps. Smaller spatial scales are probed using dust continuum emission maps from the SMA that were obtained at about $280 \mathrm{GHz}$ frequency. Specifically we observed the following targets: $\operatorname{Sgr}$ C, $20 \mathrm{~km} \mathrm{~s}^{-1}$ cloud, $50 \mathrm{~km} \mathrm{~s}^{-1}$ cloud, G0.253+0.016, Sgr B1-off, and Sgr D. The maps are used to essentially obtain masses within apertures of varying size which we define via contours of constant column density (see Kauffmann et al. 2010a for details). For reference we characterize the Orion A molecular cloud in the same fashion.

The results of these measurements are shown in Fig. 1(a). On spatial scales $\sim 5$ pc, the masses - and therefore densities — of CMZ molecular clouds exceed the one of Orion A by an order of magnitude and more. However, CMZ cloud fragments of size $\sim 0.1 \mathrm{pc}$ are typically less massive than the column density peak associated with Orion KL. This means that, compared to Orion, CMZ molecular clouds are less efficient in concentrating a large fraction of the total mass into structures of small spatial scale.

Figure 1(b) provides an alternative view at the data. Here we present the mass-size slopes, $b=\mathrm{d} \ln (m) / \mathrm{d} \ln (r)$, as a function of the maximum mass per Herschel beam. 


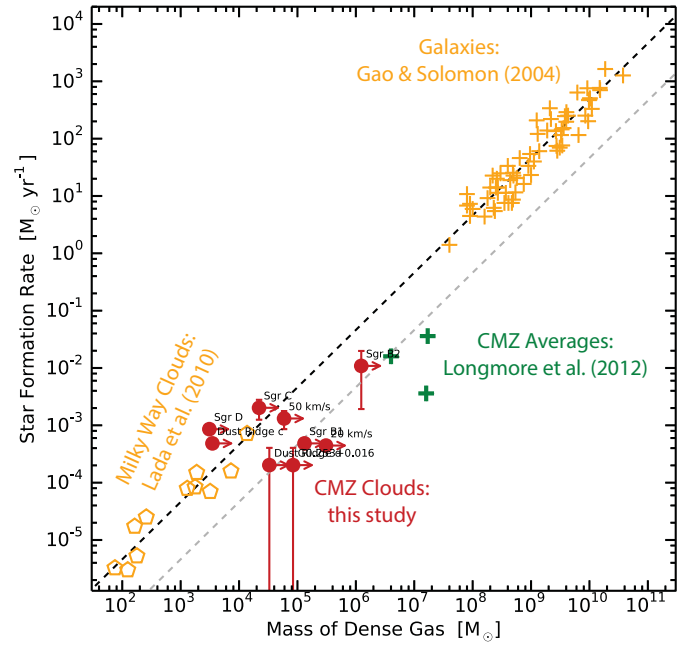

Figure 2. SF rate of molecular clouds as a function of the mass of dense gas. Note that many CMZ molecular clouds fall below the line supposedly describing regular Milky Way clouds and galaxies. (A color version of this figure is available online.)

In Kauffmann et al. (2010b) we show that $\varrho \propto r^{3-b}$ holds for singular power-law density profiles. The density laws indicated in Fig. 1(b) reveal that CMZ molecular clouds have unusually flat density profiles, compared to SF regions in the solar neighborhood (Kauffmann et al. 2010b).

In Kauffmann et al. (2013) we already revealed such a density profile in the cloud $\mathrm{G} 0.253+0.016$. Now we can assume that these flat density gradients are a general feature of many but not all CMZ molecular clouds. This absence of dense cores that could form stars likely explains the suppression of SF.

\section{CMZ Shocks revealed by ALMA}

In Kauffmann et al. (2013) we speculated that the formation of high-mass cores might be prevented by high-velocity shocks. We use ALMA observations of G0.253+0.016 to explore this picture near $220 \mathrm{GHz}$ frequency. Emission from $\mathrm{SiO}$ is usually considered to be a tracer of regions where gas streams collide at relative velocities $\gtrsim 20 \mathrm{~km} \mathrm{~s}^{-1}$. Our data reveal thin $\mathrm{SiO}$ arcs in $\mathrm{G} 0.253+0.016$, which we take to be shock fronts pervading the cloud. Further, ratios between $\mathrm{H}_{2} \mathrm{CO}$ lines indicate that the gas temperature varies between about $40 \mathrm{~K}$ and $\geqslant 200 \mathrm{~K}$ within G0.253+0.016. These temperature variations within a given cloud are most easily explained by shock heating which will induce a spatially dependent heating term.

We take this as further evidence that shocks shape CMZ molecular clouds. Numerical simulations must now show to what extent such shocks might help to suppress the fragmentation of clouds into high-mass cores. Also, it is not clear how the strong and numerous shocks in the CMZ are induced.

\section{Star Formation Activity}

Lada et al. (2010) suggest that about $5 M_{\odot}$ of gas residing at visual extinctions $A_{V} \geqslant$ 7 mag are needed to form one young stellar object. This is an important observation since this trend might also explain the supposed extragalactic correlation between dense gas and SF proposed by Gao \& Solomon (2004), as indicated in Fig. 2. We have compiled SF rates to explore this trend in the CMZ. Based on SF rates from radio-continuum emission revealing HiI regions, and dense gas masses derived from Herschel dust emission data, 


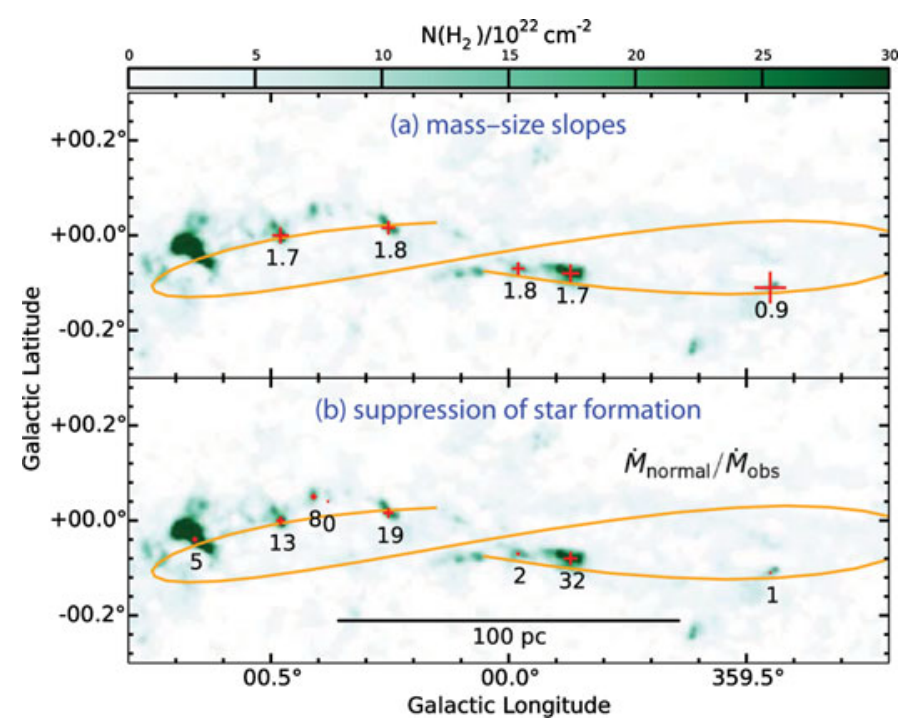

Figure 3. The orange line indicates the orbit proposed by (Kruijssen et al. 2014). No or only modest evolution is evident in density structure and SF activity. (A color version of this figure is available online.)

many CMZ molecular clouds have lower SF rates per unit dense gas than observed by Lada et al. (2010) and Gao \& Solomon (2004), as shown in Fig. 2.

\section{Evolution along Orbit}

Longmore et al. (2013) suggest that clouds evolve along the orbit identified by Molinari et al. (2011) and Kruijssen et al. (2014), for example due to compression events triggered by passages close to Sgr A*. The data collected here can be used to evaluate that picture.

Figure 3(a) presents the mass-size slopes derived from our SMA maps and Herschel data. No evolution is seen in the data. However, we caution that the current data set only covers a fraction of the CMZ. Figure 3(b) indicates the factor by which the observed SF rate in a CMZ cloud, $\dot{M}_{\text {obs }}$ is below the SF rate expected for Milky Way clouds based on the Lada et al. (2010) analysis, $\dot{M}_{\text {normal }}$. A modest and non-monotonous increase in SF activity might be evident at positive longitudes.

\section{References}

Gao, Y. \& Solomon, P. M. 2004, ApJ, 606, 271

Guesten, R. \& Downes, D. 1983, A\&A, 117, 343

Kauffmann, J., Pillai, T., Shetty, R., Myers, P. C., \& Goodman, A. A. 2010a, ApJ, 712, 1137

-. 2010b, ApJ, 716, 433

Kauffmann, J., Pillai, T., \& Zhang, Q. 2013, ApJ, 765, L35

Kruijssen, J. M. D., Dale, J. E., \& Longmore, S. N. 2014, MNRAS, 447, 1059

Lada, C. J., Lombardi, M., \& Alves, J. a. F. 2010, ApJ, 724, 687

Lis, D. C., Menten, K. M., Serabyn, E., \& Zylka, R. 1994, ApJ, 423, L39

Longmore, S. N., Bally, J., Testi, L., et al. 2012, MNRAS, 429, 987

Longmore, S. N., Kruijssen, J. M. D., Bally, J., et al. 2013, MNRAS, 433, L15

Molinari, S., Bally, J., Noriega-Crespo, A., et al. 2011, ApJ, 735, L33

Morris, M. \& Serabyn, E. 1996, ARAA, 34, 645 